\title{
Tecnologias cuidativo-educacionais para o cuidado domiciliar de crianças em uso de traqueostomia: revisão integrativa
}

\author{
Care-educational technologies to home care of children that tracheostomy: integrative review \\ Tecnologías cuidado-educativas para la asistencia a domicilio de niños en uso de traqueostomía: \\ revisión integradora
}

Recebido: 28/12/2021 | Revisado: 06/01/2022 | Aceito: 15/01/2022 | Publicado: 17/01/2022

\author{
Diana Augusta Tres \\ ORCID: https://orcid.org/0000-0001-8983-3727 \\ Universidade do Estado de Santa Catarina, Brasil \\ E-mail: dianaa.tres@gmail.com \\ Franklin de Almeida Cipolato \\ ORCID: https://orcid.org/0000-0002-4345-6740 \\ Universidade do Estado de Santa Catarina, Brasil \\ E-mail: franklincipolato1999@ hotmail.com \\ Eduarda da Silveira Castro \\ ORCID: https://orcid.org/0000-0003-3942-8760 \\ Universidade do Estado de Santa Catarina, Brasil \\ E-mail: eduarda.castro@edu.udesc.br \\ Camila Uberti \\ ORCID: https://orcid.org/0000-0002-5030-7874 \\ Universidade do Estado de Santa Catarina, Brasil \\ E-mail: camilauberti0@gmail.com \\ Rafael Gue Martini \\ ORCID: https://orcid.org/0000-0002-7409-4340 \\ Universidade do Estado de Santa Catarina, Brasil \\ E-mail: rafael.martini@udesc.br \\ Beatriz Rosana Gonçalves de Oliveira Toso \\ ORCID: https://orcid.org/0000-0001-7366-077X \\ Universidade Estadual do Oeste do Paraná, Brasil \\ E-mail: 1b.toso@gmail.com \\ Elisangela Argenta Zanatta \\ ORCID: https://orcid.org/0000-0002-7426-6472 \\ Universidade do Estado de Santa Catarina, Brasil \\ E-mail: elisangela.zanatta@udesc.br
}

\begin{abstract}
Resumo
Introdução: as tecnologias cuidativo-educacionais constituem a união da educação e do cuidado e se caracterizam pelo desenvolvimento de novas maneiras de ensinar, buscando a construção de relações entre os envolvidos, com a finalidade de promover o empoderamento, vínculo, autonomia e bem-estar de pacientes, familiares e profissionais. Objetivo: identificar que tecnologias cuidativo-educacionais podem auxiliar na capacitação de pais e cuidadores para realizar cuidados com a cânula de traqueostomia de crianças no domicílio. Método: revisão da literatura do tipo integrativa, realizada por pares, nas seguintes bases: Scopus, Biblioteca Virtual de Saúde, Publisher Medline e no catálogo de teses e dissertações da Coordenação de Aperfeiçoamento de Pessoal de Nível Superior em março de 2021, com recorte temporal de dez anos, seguindo as recomendações do método Preferred Reporting Items for Systematic Reviews and Meta-Analyses. Resultados: foram identificados 1.535 estudos, após empregar os critérios de inclusão e exclusão restaram 495 estudos; em seguida, a partir da leitura dos títulos e/ou resumos foram selecionados 31 artigos, sendo a amostra final composta por sete artigos, um nacional e seis internacionais. Foram identificadas seis tecnologias cuidativo-educacionais: simulação, demonstração, materiais impressos, materiais audiovisuais, protocolos e roda de conversa. A maioria dos estudos descreveu o uso concomitante de duas ou mais tecnologias para auxiliar nos cuidados. Conclusão: as tecnologias cuidativo-educacionais mostraram-se inovadoras, completas e objetivas, evidenciando a participação ativa do enfermeiro nas ações de educação em saúde.
\end{abstract}

Palavras-chave: Assistência domiciliar; Tecnologia educacional; Enfermagem; Traqueostomia; Assistência integral à saúde da criança.

\section{Abstract}

Background: care-educational technologies are the union of education and care and are charactized by the development of new ways of teaching, searching for relationship building among those involved, with the aim to 
promote empowerment, bond, autonomy and well-being of patients, relatives and profissionals. Objective: to identify the care-educational technologies that can help with training of relatives and caretakers to make the tracheostomy tube's care of children at home. Methods: It is treated of an integrative literature review made like peer review the databases of Scopus, the Virtual Health Library, Publisher Medline and at theses and dissertations's catalogue of Coordination for the Improvement of Higher Education Personnel in March 2021, limiting to studies published in the last ten years, following the recommendations of Method of Preferred Reporting Items for Systematic Reviews and Meta-Analyses. Results: it has been identified 1535 studies, to which it was applied the inclusion and exclusion the remaining 495 studies; therefore, from the reading of titles and/or abstracts, 31 articles were selected from which of the seven articles constituted the final sample: one national and six international. Six care-educational technologies have been identified: simulation, demonstration, printed materials, audiovisual materials, protocols and round of conversation. Most studies described the concomitant use of two or more technologies to aid in care. Conclusions: the care-educational technologies proved to be innovative, complete and objective, highlighting the nurse's active participation on actions of health education.

Keywords: Home nursing; Educational technology; Nursing; Tracheostomy; Comprehensive child health care.

\section{Resumen}

Introducción: las tecnologías cuidado-educativas constituyen la unión de la educación y del cuidado y se caracterizan por el desarrollo de nuevas formas de enseñanza, buscando crear relaciones entre los involucrados, con la finalidad de promover el apoderamiento, la vinculación, la autonomía y el bienestar de los pacientes, familiares y profesionales. Objetivo: identificar qué tecnologías de educación asistencial pueden ayudar en la formación de los padres y cuidadores en la promoción del cuidado com el tubo de traqueostomía para niños en domicilio. Método: revisión integradora de la literatura, realizada por pares, sobre las siguientes bases: Scopus, Biblioteca Virtual de Saúde, Publisher Medline y en el catálogo de tesis y disertaciones de la Coordenação para o Aperfeiçoamento de Pessoal de Nível Superior en marzo de 2021, con un marco temporal de diez años, siguiendo las recomendaciones del método Preferred Reporting Items for Systematic Reviews and Meta-Analyses. Resultados: se identificaron 1535 estudios, tan pronto a utilizar los criterios de inclusión y exclusión, quedaron 495 estudios; luego, a partir de la lectura de los títulos y/o resúmenes, se seleccionaron 31 artículos, siendo la muestra final compuesta por siete artículos, uno nacional y seis internacionales. Se identificaron seis tecnologías cuidado-educativas: simulación, demostración, material impreso, material audiovisual, protocolos y círculo de conversación. La mayoría de los estudios describieron el uso congruente de dos o más tecnologías para ayudar en la atención. Conclusión: las tecnologías cuidado-educativas resultaron ser innovadoras, completas y objetivas, mostrando la participación activa del enfermero en las acciones de educación em salud.

Palabras clave: Atención domiciliaria de salud; Tecnología educacional; Enfermería; Traqueostomía; Atención integral de salud infantil.

\section{Introdução}

Os avanços científicos e tecnológicos têm provocado mudanças importantes na atenção à saúde da criança, especialmente, no que diz respeito à melhoria no acesso aos serviços de saúde, a qualidade no atendimento de pré-natal e a assistência à saúde durante os primeiros anos de vida, levando ao aumento da sobrevivência de crianças que antes morriam precocemente devido a doenças graves como prematuridade, malformações congênitas e condições crônicas (Carvalho et al., 2019; Kirchchoff et al., 2020).

No Brasil, o grupo das crianças que apresentam condições crônicas de saúde e necessitam do uso de dispositivos tecnológicos para sobreviver são denominadas Crianças com Necessidades Especiais de Saúde (CRIANES), termo traduzido e adaptado da língua inglesa, Children with Special Health Care Needs (CSHCN) (Arrué et al., 2016; McPherson et al., 1998).

A Organização Mundial da Saúde (OMS), no final da década de 90, mudou a definição de doenças crônicas para condições crônicas. Assim, essa nova nomenclatura abrange diversas patologias como diabetes, doenças cardiovasculares, câncer, doenças respiratórias crônicas, doenças musculoesqueléticas, doenças infecciosas persistentes, condições ligadas à maternidade, ao período perinatal e à manutenção da saúde por ciclos de vida, distúrbios mentais de longo prazo, deficiências físicas e estruturais contínuas, doenças metabólicas e doenças bucais não agudizadas (Carvalho et al., 2019; Mendes, 2012).

Nesse contexto, a complexidade foi inserida nas condições crônicas, dando origem ao termo Condições Crônicas Complexas (CCCs), que se refere a presença de uma condição crônica, associada a comorbidades de diferentes graus e a utilização de serviços hospitalares. As principais características das CCCs envolvem a necessidade de tratamento contínuo e de 
longa duração, limitações funcionais, necessidades de cuidados especializados e dependência tecnológica (Carvalho et al., 2019; Moura et al., 2017).

Dentre as pessoas com CCCs, destacam-se as CRIANES que se caracterizam por apresentarem uma ou mais condição crônica de saúde ou possuírem um risco maior para desenvolvê-las. Essas crianças, normalmente, necessitam de um cuidado contínuo, temporário ou, muitas vezes, permanente e de um número maior de atendimentos do que uma criança que não apresente uma condição crônica.

Conhecer o número de CRIANES com CCCs é importante para que os serviços de saúde possam planejar ações direcionadas à assistência desse público, contudo, no cenário de saúde brasileiro não existem dados epidemiológicos atuais. O último registro nacional foi realizado no ano de 2010, pelo Instituto Brasileiro de Geografia e Estatística (IBGE) revelando que 9,1\% das crianças de zero a cinco anos, 9,7\% das crianças de seis a 13 anos e $11 \%$ dos adolescentes de 14 a 19 anos possuem uma condição crônica de saúde (Rossetto et al., 2019). Além da falta de dados, De Sousa Lima et al., (2021) e Rossetto et al., (2019) discutem acerca da ausência de políticas públicas específicas para as CRIANES, evidenciando a pouca atenção voltada a esse grupo que necessita de cuidados constantes e, por vezes, complexos.

Os cuidados demandados pelas CRIANES são divididos em seis categorias: a primeira inclui crianças com atraso de desenvolvimento, na sequência estão aquelas que necessitam de cuidados com dispositivos tecnológicos, na terceira as que fazem uso contínuo de fármacos, na quarta estão as crianças que precisam de adaptações nas atividades rotineiras, na quinta as que possuem uma ou mais das demandas anteriores, excluindo-se a tecnológica e a sexta categoria engloba as crianças com demandas de cuidados clinicamente complexos, possuem todas as anteriores inclusive o manejo de tecnologias para suporte à vida (Góes \& Cabral, 2017).

Considerando essa classificação, o presente estudo tem como foco a segunda e a sexta categorias, nas quais encontram-se as crianças que necessitam de cuidados tecnológicos, especificamente, as que fazem uso do dispositivo de traqueostomia no domicílio.

A evolução tecnológica permite a transferência do cuidado de crianças do ambiente hospitalar para o domiciliar, contudo a família que passará por esse processo de desospitalização, precisa ser assistida por todos os serviços que compõe a Rede de Atenção à Saúde (RAS), para que se sinta segura e amparada no momento de levar a criança para casa (Carvalho et al., 2019).

A desospitalização compreende a alta hospitalar precoce de forma segura e responsável, o que requer planejamento detalhado e rigoroso que envolve a realização de ações educativas com familiares e cuidadores para o manejo e cuidados específicos conforme a necessidade de cada criança. Os familiares, na maioria dos casos, passam a ser responsáveis por realizar o cuidado com a criança dependente de tecnologia no domicílio. Desse modo, considerando a complexidade dos cuidados demandados pela criança, após a alta hospitalar, exige-se que os pais adquiram conhecimentos e habilidades específicas para desenvolver essa tarefa no domicílio. Assim, o saber da enfermagem precisa ser compartilhado e ensinado, visto a necessidade de orientações e capacitações exigidas para uma assistência adequada (Carvalho et al., 2019; Kirchchoff et al., 2020; Bossa et al., 2019).

Dessa forma, a utilização de Tecnologias Cuidativo-Educacionais (TCE) voltadas aos cuidados vem crescendo no meio científico e são consideradas fundamentais para subsidiar uma assistência segura. Elas favorecem o processo de cuidado e contribuem para que os profissionais incentivem o pensamento crítico dos familiares e cuidadores (Moreira et al., 2017).

O conceito de TCE, reconstruído a partir de ações de enfermagem, constituí a união da educação e do cuidado. O processo de cuidar-educar e educar-cuidar em enfermagem estão sempre interligados, buscando a construção de relações entre os envolvidos, com a finalidade de promover o empoderamento, vínculo, autonomia e bem-estar de pacientes, familiares e profissionais (Salbego et al., 2018). 
Considerando as necessidades da criança em uso de traqueostomia, sobretudo em relação aos cuidados demandados por esse dispositivo, o objetivo deste estudo foi identificar que Tecnologias Cuidativo-Educacionais podem auxiliar na capacitação de pais e cuidadores para realizar os cuidados com a cânula de traqueostomia de crianças no domicílio.

\section{Metodologia}

Trata-se de uma revisão integrativa (RI), desenvolvida a partir de um protocolo que contemplou os seguintes passos: 1) definição da equipe de pesquisadores; 2) identificação da questão de pesquisa; 3) avaliação do protocolo; 4) seleção e extração dos estudos; 5) validação da seleção dos estudos incluídos; 6) avaliação e análise dos estudos incluídos; 7) análise e interpretação dos resultados da revisão (Zocche et al., 2020).

A questão de pesquisa foi elaborada a partir da estratégia: PICO, sendo População (cuidadores), Intervenção (uso de TCE), Conduta (atividade educativa/capacitação), Outcomes (melhores TCE), ficando com a seguinte redação: que Tecnologias Cuidativo-Educativas podem auxiliar na capacitação de pais e cuidadores para a realização dos cuidados com a cânula de traqueostomia de crianças no domicílio?

Os critérios de inclusão foram: artigos, teses e dissertações disponíveis on-line, nos idiomas português, inglês e espanhol, publicados de 2011 a março de 2021. Esse recorte temporal foi definido pelo ano de implantação da Atenção Domiciliar (AD) no âmbito do Sistema Único de Saúde (SUS), portaria no 2.029 de 24 de agosto de 2011 (de Oliveira Neto \& Borges Dias, 2014).

Foram excluídas revisões integrativas, narrativas e sistemáticas, documentários, resenhas, editoriais, documentos legais e artigos duplicados.

A pesquisa ocorreu no mês de março de 2021, por pares. Foi realizada na Scopus, Biblioteca Virtual de Saúde (BVS), Publisher Medline (PubMed) e no catálogo de teses e dissertações da Coordenação de Aperfeiçoamento de Pessoal de Nível Superior (CAPES), utilizando-se os seguintes descritores e estratégias de cruzamento: (1) Serviços de Assistência Domiciliar AND Enfermagem AND Traqueostomia AND Criança; (2) Tecnologia educacional (AND) Assistência Domiciliar AND Traqueostomia.

Foram identificados 1.534 artigos e uma dissertação, após aplicar os critérios de inclusão e exclusão restaram 495 estudos, dos quais foram lidos os títulos e os resumos. Após essa leitura foram excluídos 464, restando 31 estudos que foram lidos na íntegra. Após a leitura na íntegra, foram eleitos sete artigos que passaram a compor a amostra, conforme fluxograma construído seguindo a metodologia Preferred Reporting Items for Systematic Reviews and Meta-Analyses PRISMA (Galvão et al., 2015) apresentado na Figura 1. 
Figura 1. Diagrama PRISMA dos estudos selecionados.

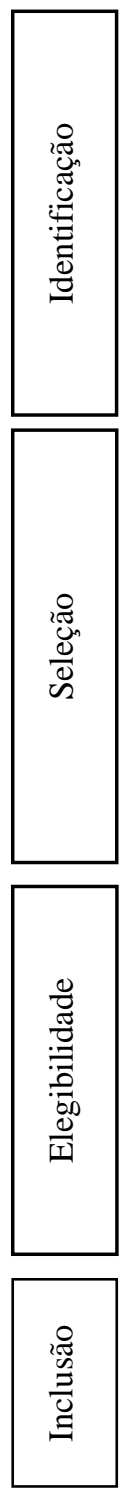

Estudos identificados $(\mathrm{n}=1.535)$

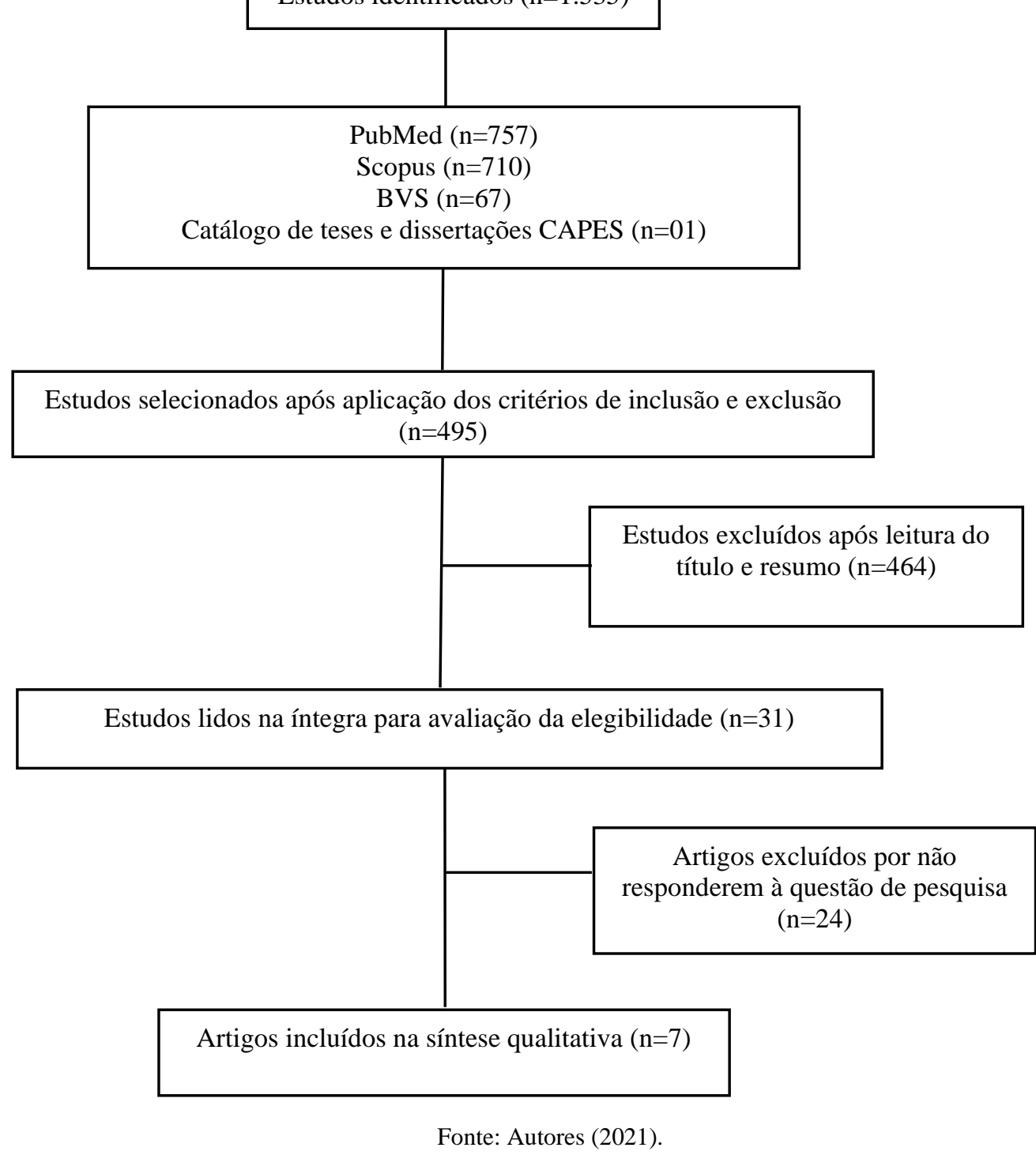

Após a seleção dos artigos e leitura crítica, foi preenchida uma planilha para a análise integrativa e qualitativa de caráter básico e extração das informações visando responder à questão de pesquisa e o objetivo do estudo (Estrela, 2018).

\section{Resultados}

Os artigos incluídos nessa RI apresentam TCE relacionadas ao cuidado à criança em uso de traqueostomia. Para compor a amostra final, foram selecionados sete (100\%) artigos, dos quais cinco $(71,4 \%)$ na base de dados Scopus, um $(14,2 \%)$ na PubMed e um (14,2\%) na BVS. Os estudos foram publicados entre 2013 e 2018, sendo a maioria (57,1\%) em 2016.

Dos artigos analisados, seis $(85,7 \%)$ foram publicados em periódicos internacionais e apenas um em periódico nacional. No idioma inglês, cinco $(71,4 \%)$, espanhol um $(14,2)$ e português um $(14,2 \%)$. Em relação aos países de desenvolvimento e publicação dos estudos, houve a seguinte distribuição: Estados Unidos da América quatro (57,1\%), Brasil um (14,2\%), Argentina um (14,2\%) e Nova Zelândia um (14,2\%).

No Quadro 1 estão apresentados os sete artigos selecionados, considerando o título, autores, ano de publicação, país de publicação, TCE e nível de evidência (Ribeiro \& Aroni, 2019). 


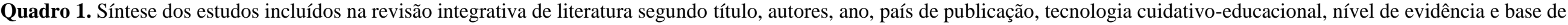
dados. Chapecó, Santa Catarina, 2021.

\begin{tabular}{|c|c|c|c|c|c|}
\hline Título & Autores & Ano & $\begin{array}{c}\text { País de } \\
\text { publicação }\end{array}$ & Tecnologia Cuidativo-Educacional & Nível de Evidência \\
\hline \multicolumn{6}{|c|}{ Scopus } \\
\hline $\begin{array}{l}\text { A1 - Encontro educativo da enfermagem e da } \\
\text { família de crianças com necessidades especiais } \\
\text { de saúde. }\end{array}$ & $\begin{array}{l}\text { Viana IS, Da Silva LF, } \\
\text { Cursino EG; Da Conceição } \\
\text { DS, Goes FGB; De Moraes } \\
\text { JRMM. }\end{array}$ & 2018 & Brasil & $\begin{array}{l}\text { Roda de conversa, com demonstração em um boneco, dos cuidados } \\
\text { com dispositivos tecnológicos (cânula de traqueostomia, cateter de } \\
\text { gastrostomia, cateter nasogástrico, cateter nasal para oxigenoterapia e } \\
\text { bolsa de colostomia). }\end{array}$ & Nível IV \\
\hline $\begin{array}{l}\mathrm{A} 2 \text { - Becoming an expert carer: the process of } \\
\text { family carers learning to manage technical } \\
\text { health procedures at home. }\end{array}$ & $\begin{array}{l}\text { McDonald J, McKinlay E, } \\
\text { Keeling S, Levack W. }\end{array}$ & 2016 & Nova Zelândia & Simulação e material impresso. & Nível IV \\
\hline $\begin{array}{l}\text { A3 - Identification of fail points for } \\
\text { discharging pediatric patients with new } \\
\text { tracheostomy and ventilator. }\end{array}$ & $\begin{array}{l}\text { Sobotka SA, Hird-McCorry } \\
\text { LP, Goodman DM. }\end{array}$ & 2016 & $\begin{array}{l}\text { Estados Unidos da } \\
\text { América }\end{array}$ & $\begin{array}{l}\text { Programa de Habilitação Pulmonar (PHP) - Simulação (consiste em } \\
\text { treinar e preparar cuidadores para o cuidado de uma criança ventilada } \\
\text { em casa). }\end{array}$ & Nível IV \\
\hline $\begin{array}{l}\mathrm{A} 4-\text { Preventing complications of pediatric } \\
\text { tracheostomy through standardized wound care } \\
\text { and parent education. }\end{array}$ & $\begin{array}{l}\text { Gaudreau PA, Greenlick H, } \\
\text { Dong T, Levy M, Hackett A, } \\
\text { Preciado D, et al. }\end{array}$ & 2016 & $\begin{array}{l}\text { Estados Unidos da } \\
\text { América }\end{array}$ & $\begin{array}{l}\text { Protocolo para educação do cuidador "Trach Me Home": simulação e } \\
\text { vídeo. }\end{array}$ & Nível IV \\
\hline $\begin{array}{l}\text { A5 - A standardized discharge process } \\
\text { decreases length of stay for ventilator- } \\
\text { dependent children. }\end{array}$ & $\begin{array}{l}\text { Christopher D. Baker, Sara } \\
\text { Martin, Jodi Thrasher, } \\
\text { Heather M. Moore, Joyce } \\
\text { Baker, Steven H. Abman, } \\
\text { Jason Gien. }\end{array}$ & 2016 & $\begin{array}{l}\text { Estados Unidos da } \\
\text { América }\end{array}$ & Roteiro de ventilação crônica: Simulação, vídeos e material impresso. & Nível II \\
\hline \multicolumn{6}{|c|}{ Pubmed } \\
\hline $\begin{array}{l}\text { A6 - Hospital to home: a quality improvement } \\
\text { initiative to implement high-fidelity simulation } \\
\text { training for caregivers of children requiring } \\
\text { long-term mechanical ventilation. }\end{array}$ & $\begin{array}{l}\text { Thrasher J, Baker J, Ventre } \\
\text { KM, Martin SE, Dawson J, } \\
\text { Cox R, et al. }\end{array}$ & 2017 & $\begin{array}{l}\text { Estados Unidos da } \\
\text { América }\end{array}$ & $\begin{array}{l}\text { Programa de treinamento para a alta: Simulação, vídeos e material } \\
\text { impresso. }\end{array}$ & Nível IV \\
\hline \multicolumn{6}{|c|}{ BVS } \\
\hline $\begin{array}{l}\text { A7 - Gestion de cuidados de enfermeria de los } \\
\text { niños internados con traqueostomia. } \\
\text { Experiencia en el CIM 62. Cambio de cánula } \\
\text { de traqueostomía. Protocolo de entrenamiento } \\
\text { para padres y cuidadores. Experiencia en el } \\
\text { Hospital Garrahan. }\end{array}$ & Rivas E, Peña M, Silva A. & 2013 & Argentina & Protocolo de treinamento para pais: simulação e material impresso. & Nível IV \\
\hline
\end{tabular}

Fonte: autores (2021) 
A leitura do material selecionado permitiu identificar que as principais tecnologias utilizadas para a capacitação de pais e cuidadores no cuidado à criança em uso de traqueostomia foram: simulação, demonstração de técnicas, material impresso, materiais audiovisuais, protocolos e roda de conversa. Sendo que, a maioria dos estudos descreveu a utilização de duas ou mais tecnologias, utilizadas de forma concomitante para atingir o objetivo proposto. Assim, a simulação foi utilizada em seis $(84,7 \%)$ dos estudos, o material impresso em quatro $(57,1 \%)$, o material audiovisual em três $(42,8)$, o protocolo em dois $(28,5 \%)$, a roda de conversa e a demonstração em somente um $(14,2 \%)$ dos estudos.

\section{Discussão}

As TCE se caracterizam pelo desenvolvimento de novas teorias, pesquisas, conceitos, técnicas e maneiras de ensinar, proporcionado ao educador a atualização e inovação na forma de compartilhar o conhecimento com o educando, com isso, facilitando e contribuindo com o seu aprendizado. A utilização de TCE pelos enfermeiros proporciona o aperfeiçoamento da prática do cuidado e das ações de atenção à saúde, auxiliando e emponderando os sujeitos envolvidos durante os processos de educação e cuidado (Salbego et al., 2018).

O cenário atual de cuidados em saúde revela que, cada vez mais, a realização de procedimentos complexos, que seriam restritos aos profissionais de saúde em um ambiente hospitalar, como alimentação por sonda enteral, terapia intravenosa, diálise e cuidados com a traqueostomia, estão sendo realizados em casa por familiares e cuidadores. Num cenário ideal, os cuidados domiciliares deveriam ser prestados por profissionais capacitados. Entretanto, essa não é a realidade nacional, o que demanda dos profissionais de saúde atividades de capacitação para os cuidadores familiares. Diante disso, os profissionais de saúde precisam, inicialmente, avaliar a capacidade de compreensão, aprendizagem e necessidades dos cuidadores no ambiente doméstico (McDonald et al., 2016). Após esse reconhecimento, poderão planejar e escolher a TCE que melhor se adapte a cada realidade.

Na presente pesquisa, constatou-se escassa a produção acerca do objetivo do estudo. Em um período de dez anos foram identificados, nas bases pesquisadas, apenas sete estudos que apresentavam e discutiam sobre TCE utilizadas para capacitação de pais e cuidadores de crianças em uso de traqueostomia no domić́lio, desses, apenas um estudo brasileiro. Talvez, esse resultado possa ser explicado pelo número reduzido de pesquisas relacionadas a esse tema, associado a pouca disponibilidade dos serviços, pois dos 5.570 municípios brasileiros, somente 610 contam com o Serviço de Atenção Domiciliar (SAD) implantado. Além disso, não se tem informações sobre o número de CRIANES em uso de traqueostomia, acompanhadas por eles e, nem os recursos utilizados para a capacitação de pais e cuidadores para o cuidado domiciliar a essas crianças (Brasil, 2020).

Nesse contexto, no único estudo brasileiro A1, a TCE utilizada para a capacitação de pais e cuidadores de CRIANES, foi uma roda de conversa, aliada a demonstração de cuidados em um boneco que possuía acoplado a seu corpo, dispositivos tecnológicos, dentre eles a cânula de traqueostomia. Essa estratégia foi utilizada por enfermeiros que atuavam em um hospital pediátrico e identificaram a necessidade de capacitar os pais para os cuidados com esse dispositivo. Assim, organizaram rodas de conversa para discutir as dificuldades e a partir delas demonstrar os cuidados com os dispositivos. Após a demonstração, os participantes do estudo manusearam o boneco e os aparatos tecnológicos inseridos nele (Viana et al., 2018).

O uso da roda de conversa nos processos de educação em saúde, favorece o diálogo entre os participantes, permitindo que todos expressem suas opiniões, impressões e concepções acerca de determinada temática. A interação social, por meio de diálogos e debates, torna a roda de conversa um recurso didático criativo e eficaz, sendo compreendida como um espaço amplo de cuidado, contribuindo para a execução de novas formas de fazer saúde (Ferreira \& Abrahão, 2020; Barbosa Amorim et al., 2020). 
Desse modo, a participação ativa proporcionada pela roda de conversa, ajuda os envolvidos no processo de ensino/aprendizagem e pode trazer benefícios para a autonomia e autocuidado de cada participante. Na comunicação em grupo, o compartilhamento de vivências e experiências sobre determinado assunto, permite a produção de conteúdo e possibilita a elaboração de estratégias para a atenção integral à saúde da população (Dias et al., 2018).

Sendo assim, acredita-se que o uso da roda de conversa como TCE para a realização de uma atividade prática que envolve a demonstração dos cuidados, tem o propósito de estimular a participação e a interação entre os participantes, oportunizando a troca de experiências e vivências acerca do cuidado com o dispositivo de traqueostomia.

No estudo A3, a tecnologia utilizada foi a simulação, desenvolvida em um Hospital Infantil de Chicago, como parte do Programa de Habilitação Pulmonar (PHP), cujo objetivo foi preparar os pais para cuidar da criança em casa. O programa preconiza o ensino completo de um cuidador primário e um secundário, por meio de capacitações que iniciam no ambiente hospitalar e pode incluir cuidados com a traqueostomia, sonda para alimentação, cuidados com a administração de medicações, gerenciamento de ventilador mecânico, ressuscitação cardiopulmonar e intervenções de emergência. Antes de a criança receber alta hospitalar, os cuidadores precisam demonstrar o cuidado com a criança de forma independente por 24 horas consecutivas (Sobotka et al., 2016).

Os estudos A2, A4, A5, A6, e A7 apresentam a simulação associada a outras TCE para capacitação de pais e cuidadores na realização do cuidado domiciliar à criança em uso de traqueostomia. $\mathrm{O}$ estudo A2 utilizou simulação e material impresso, A4 discute o uso de um protocolo que contempla a simulação associada a vídeos, A5 e A6 utilizaram simulação de alta fidelidade, vídeos e material impresso e A7 também desenvolveu um protocolo para a educação em saúde com o uso da simulação e material impresso.

Em A2, o enfermeiro, após realizar a explicação associada à demonstração solicitava que os cuidadores realizassem cuidados, como administração de dieta, dosagem de glicose, realização do cateterismo urinário intermitente, aspiração da cânula de traqueostomia, curativos em feridas, dentre outros. Os cuidadores praticavam em seus familiares, em bonecos de simulações e em si mesmos, a prática assistida permitindo que o enfermeiro observasse se o procedimento estava sendo executado com competência, proporcionando que o cuidador obtivesse o feedback sobre o seu desempenho na realização dos cuidados com os dispositivos. Além da atividade prática, os cuidadores também receberam material impresso sobre o procedimento que aprenderam (McDonald et al., 2016).

O uso da simulação, associada aos vídeos, também foi a tecnologia utilizada em A4, sendo descrita a utilização do protocolo "Trach Program Me Home" (TMH) desenvolvido em 2011, no Children's National Medical Center (CNMC) de Washington, para subsidiar a educação de pais e cuidadores de crianças em uso de traqueostomia. Segundo o protocolo, a capacitação é iniciada ainda no pré-operatório com um vídeo de introdução sobre o dispositivo. No pós-operatório, os pais assistiam vídeos sobre temas relacionados a limpeza da traqueostomia, troca do cadarço, avaliação da pele periestoma, sons emitidos pela traqueostomia, aspiração da cânula de traqueostomia com luvas esterilizadas, procedimentos de emergência (obstrução, decanulação e sangramento) e demonstração de ressuscitação cardiopulmonar (RCP) com uso do reanimador manual conectado na traqueostomia. Após, os cuidadores praticavam os cuidados com a traqueostomia em uma boneca, incluindo a troca da cânula (Gaudreau et al., 2016).

A5 apresenta como tecnologia educativa um roteiro de ventilação crônica denominada de "Chronic Ventilation Road Map" desenvolvida por profissionais de um Hospital Infantil do Colorado, com o objetivo de descrever o passo a passo para o planejamento da alta e capacitação de pais e cuidadores de crianças dependentes de ventilação mecânica, via traqueostomia. A atividade com os pais e cuidadores era realizada em um laboratório de simulação de alta fidelidade e incluía os seguintes cuidados com a traqueostomia: aspiração e troca da cânula, ventilação na traqueostomia com uso do reanimador manual e 
reanimação cardiopulmonar. Os profissionais também disponibilizavam material impresso e vídeos para auxiliar na educação de pais e cuidadores e prepará-los para a alta da criança (Baker et al., 2016).

A simulação de alta fidelidade associada a vídeos e materiais impressos, também é descrita em A6. Nesse estudo, o cenário foi um hospital infantil de cuidados terciários e a tecnologia desenvolvida foi um programa de capacitação para a alta, composto por folhetos impressos, vídeos instrutivos, atividade prática à beira do leito, simulação de alta fidelidade de traqueostomia e uma estadia independente. A estadia independente consiste em um período de um a três dias durante a internação no qual o cuidador familiar cuida da criança de forma independente para se preparar para a alta (Thrasher et al., 2018).

Em A7, o protocolo de capacitação de pais e cuidadores para a troca da cânula de traqueostomia, foi desenvolvido pela equipe de enfermagem em conjunto com os serviços de endoscopia respiratória e unidade de terapia intensiva de um hospital infantil na Argentina. O protocolo indica que os pais e cuidadores realizem a prática simulada em um centro de treinamento no hospital, posteriormente, recebem um material impresso, do tipo folheto informativo, que reforça as orientações e os auxilia no cuidado à criança em uso de traqueostomia (Rivas et al., 2013).

$\mathrm{Na}$ área da enfermagem, o uso da simulação clínica como estratégia de ensino vem se destacando pela possibilidade de expor os estudantes a cenários hipotéticos que simulam uma realidade vivenciada na prática clínica. Nesse sentido, acreditase que o uso de estratégias práticas, como a simulação, tem grande potencial na capacitação de cuidadores domiciliares, contribuindo com o aprendizado, por permitir que o aprendiz realize os cuidados por meio da reprodução de sua situação real, possibilitando que os erros ocorram em um ambiente controlado, com isso aumentando a autoconfiança e o desenvolvimento de competências do cuidador (Orlandin et al., 2017; Prickett et al., 2019; Nascimento et al., 2021).

Assim, considerando a complexidade que é cuidar de uma criança dependente de traqueostomia no domicílio e que, na maioria das vezes, esses cuidados são realizados pelos membros da família, a capacitação com o uso da simulação proporciona uma situação realística acerca dos cuidados com esse dispositivo, possibilitando que os pais desenvolvam habilidades por meio da prática, além de vivenciarem experiências reais de simulação frente a situações de complicações e/ou emergências (Prickett et al., 2019).

O uso da simulação como TCE é evidenciada em estudo realizado com pacientes submetidos a cateterismo intermitente limpo e seus cuidadores em um centro de reabilitação de um hospital universitário do estado de São Paulo, com o objetivo de desenvolver habilidades e competências com o uso da simulação para capacitar pacientes e cuidadores para realizar o procedimento. Ao final, a estratégia educacional de simulação provou ser eficaz, pois melhorou o conhecimento e a tomada de decisões em situações iatrogênicas relacionadas ao cateterismo urinário (Orlandin et al., 2017).

Outros dois estudos também relataram o uso da simulação de alta fidelidade para capacitação de cuidadores, destacando que essa vem sendo cada vez mais utilizada na área da saúde, principalmente pelos cursos de enfermagem e em serviços de saúde para a capacitação dos profissionais (Miranda et al., 2018; Presado et al., 2018). A simulação de alta fidelidade utiliza um boneco anatômico que apresenta funções fisiológicas muito semelhantes ao ser humano, controlado por meio de um software que realiza a simulação dos sinais e sintomas, possibilitando a realização de diferentes cenários e contribuindo com o aprendizado dos participantes. Ainda, esse método proporciona o desenvolvimento do raciocínio clínico, da confiança e da integração entre a teoria e prática (Martins et al., 2012)

Sendo assim, é importante destacar que a simulação foi a TCE utilizada na maioria dos estudos encontrados nessa RI, principalmente sendo utilizada por enfermeiros e desenvolvida, ainda, no ambiente hospitalar, com o objetivo de preparar os pais e cuidadores antes mesmo da alta, para que eles se sentissem seguros e confiantes no momento de levar seu filho para casa. 
Salienta-se que a educação em saúde com o uso da simulação é uma excelente opção para a formação técnica e o conhecimento dos cuidadores, visto que ela proporciona uma maneira realista e eficaz de fornecer experiências e desenvolver habilidades, aumentando a confiança e permitindo a educação dos familiares (Prickett et al., 2019).

A segunda TCE mais relatada nos estudos foi o material impresso sendo um grande aliado dos enfermeiros nas ações de educação em saúde. No presente estudo, quatro artigos descrevem a sua utilização para a capacitação e orientação sobre os cuidados com o dispositivo de traqueostomia. Dentre os materiais impressos utilizados, têm-se as cartilhas, que são consideradas ferramentas indispensáveis para ampliar o conhecimento, a satisfação, a adesão ao tratamento e o autocuidado em diversas populações, como pessoas que convivem com doenças crônicas, idosos e, principalmente, cuidadores de crianças que fazem o uso da traqueostomia.

Uma pesquisa desenvolvida em um município da região metropolitana de Fortaleza no período de março de 2016 a dezembro de 2017, apresenta o desenvolvimento de uma cartilha pela Equipe Multiprofissional de Atenção Domiciliar (EMAD) com foco no cuidado às crianças e adolescentes acamados e que necessitavam de cuidados especializados. O desenvolvimento da cartilha se deu a partir de entrevistas realizadas com os próprios cuidadores e que tiveram como objetivo identificar o perfil clínico das crianças e adolescentes acamados, identificar o perfil socioeconômico dos cuidadores e as principais dificuldades vivenciadas pelos mesmos durante a realização do cuidado (Oliveira et al., 2020)

O estudo também discute pontos importantes para o desenvolvimento de uma cartilha, como o uso e criação de ilustrações que representem os procedimentos realizados no domicílio, consulta de livro de fundamentos de enfermagem e de procedimentos operacionais, seleção dos conteúdos e elaboração do roteiro de forma multiprofissional. Assim, entende-se que, para a criança com traqueostomia, essas estratégias trazidas são essenciais para tornar o conteúdo mais didático e de fácil compreensão aos cuidadores perante as técnicas a serem prestadas rotineiramente (Oliveira et al., 2020).

É evidente que os materiais impressos confeccionados de forma clara, simples e objetiva, poderão proporcionar aos cuidadores e/ou familiares maior participação no autocuidado, diminuindo suas inseguranças, incertezas e o desconhecimento que podem contribuir para o surgimento das intercorrências. Ainda, estudos evidenciam a eficiência do uso da cartilha educativa, reforçando que este tipo de material poderá promover mudanças significativas na aquisição de conhecimentos e desenvolvimento de competências no processo de adaptação frente as doenças crônicas sem possibilidade de cura (Rocha et al., 2019).

O uso de vídeos foi relatado em três estudos, nesse sentido, a enfermagem tem avançado na elaboração de ferramentas educacionais, como o vídeo, sendo considerado um material didático, utilizado no processo de ensino-aprendizagem em diferentes contextos educacionais. O uso do vídeo proporciona ao profissional de saúde a possibilidade de aproximar os conteúdos abordados da realidade, despertando o interesse do público a que ele se destina, proporcionado uma melhor compreensão do conteúdo abordado (Lengruber et al., 2021; Faleiros et al., 2019).

Assim como observado neste estudo, os vídeos podem ser utilizados como materiais de apoio em conjunto com outras TE, com o propósito de auxiliar nas dificuldades e esclarecer as dúvidas, contribuindo com a aprendizagem de uma maneira simples, atrativa e dinâmica (Lengruber et al., 2021; Carvalho Neto et al., 2020)

Em relação a aceitação e avaliação do vídeo educativo pelo público-alvo, em estudo realizado em uma escola de enfermagem de São Paulo, foi desenvolvido um vídeo para a sensibilização e educação da família sobre alívio da dor aguda do bebê. Depois de pronto, o material passou pela avaliação dos pais das crianças, que o consideraram como excelente pois ele apresentava informações novas, importantes e autoexplicativas que ajudavam no controle da dor (Nazario et al., 2021).

Em relação aos protocolos, descritos nos estudos A4 e A7, eles caracterizam-se por conter orientações sistematizadas, às vezes, em formato de fluxograma ou de uma matriz temporal, baseados nas diretrizes e evidências da literatura e elaborados por especialistas que os desenvolvem pensando em um grupo específico. Assim, por meio de uma assistência sistematizada, 
utilizando protocolos assistenciais, é possível conseguir um equilíbrio entre as necessidades dos clientes e a melhoria da qualidade assistencial. A padronização dos protocolos assistenciais permite a coleta, organização, análise e descrição de ideias, assim como verifica e padroniza, servindo de base para indicadores de riscos/qualidade (Silva et al., 2017).

As ações para uma efetiva transição do cuidado não são uma realidade em grande parte das instituições de saúde brasileiras, principalmente no que diz respeito à educação do cuidador para o cuidado domiciliar. De modo específico, na atenção ao cuidador e à criança, o enfermeiro é o profissional que apresenta competências para orientá-los e tomar a frente na elaboração desses protocolos objetivando facilitar os cuidados prestados e, consequentemente, tornar a vida da criança mais saudável (Santos et al., 2020).

Sendo assim, para as crianças em uso de traqueostomia em domicílio, os cuidadores podem e devem ser orientados e guiados por protocolos específicos. O preparo do cuidador de modo adequado faz parte das ações de transição do cuidado nos três pontos de atenção da RAS (serviços hospitalares, de atenção domiciliar e de atenção básica). Pensando nessa linha, após a alta hospitalar, os cuidados com a traqueostomia requerem uma adequada transferência do hospital para o domicílio e acompanhamento da criança na sua residência, visando reduzir efeitos adversos, readmissão hospitalar e utilização de serviços de emergência.

\section{Conclusão}

Este estudo revelou o uso da simulação, como principal TCE utilizada para capacitação de pais e cuidadores no cuidado a criança em uso de traqueostomia. Sendo essa utilizada ainda no ambiente hospitalar, dessa forma, contribuindo para o preparo dos cuidadores para a alta hospitalar e para o cuidado domiciliar. Do mesmo modo, a associação de duas ou mais tecnologias, revelou-se como estratégia que proporciona a complementação das informações.

A atuação do enfermeiro ficou evidente na maioria dos estudos, sendo esse o profissional que, na maioria das vezes, planeja, organiza e desenvolve as ações de educação em saúde, estimulando a participação de outros profissionais que atuam no cuidado ao público pediátrico. Nesse sentido, é essencial que o enfermeiro realize suas ações baseadas em evidências científicas, compartilhando o seu conhecimento com o restante da equipe multidisciplinar, interdisciplinar e com usuários/família/cuidadores.

As tecnologias cuidativo-educacionais apresentadas nos estudos mostraram-se inovadoras, completas e objetivas. Espera-se que com este estudo, outros serviços que realizam a assistência à criança em uso de traqueostomia, sejam estimulados para publicação de suas experiências ou até mesmo ao desenvolvimento de tecnologias voltadas para o público pediátrico, com o propósito de qualificar o cuidado prestado às CRIANES.

Do mesmo modo, sugere-se a realização de novos estudos que abordem os resultados obtidos com aplicação de tecnologias cuidativo-educacionais na capacitação de pais e cuidadores para o cuidado domiciliar às crianças em uso dispositivos tecnológicos para a manutenção da vida.

\section{Referências}

Arrué, A. M., Neves, E. T., Magnago, T. S. B. d. S., Cabral, I. E., Gama, S. G. N. d., \& Hökerberg, Y. H. M. (2016). Tradução e adaptação do Children with Special Health Care Needs Screener para português do Brasil. Cadernos de Saúde Pública, 32(6). https://doi.org/10.1590/0102-311x00130215

Baker, C. D., Martin, S., Thrasher, J., Moore, H. M., Baker, J., Abman, S. H., \& Gien, J. (2016). A Standardized Discharge Process Decreases Length of Stay for Ventilator-Dependent Children. Pediatrics, 137(4). https://doi.org/10.1542/peds.2015-0637

Barbosa Amorim, L., Rejane Santos, M., Mendes Santos, J. A., da Paz Santos, C., Dos Santos Iochims, F., \& Alves Ribeiro, W. (2020). A roda de conversa como instrumento de cuidado e promoção da saúde mental: percepção dos usuários dos CAPS. Nursing, $23(263)$, 3710-3715. https://doi.org/10.36489/nursing.2020v23i263p3710-3715 
Brasil. (2020). Dados e informações: Procedimentos SAD 2016 a 2019. Ministério da Saúde. https://antigo.saude.gov.br/acoes-e-programas/melhor-em-casaservico-de-atencao-domiciliar/dados-e-informacoes

Carvalho, M. S. N. de, Almeida de Menezes, L., Domiciano da Cruz Filho, A., \& Maurício de Paulo Maciel, C. (2019). Desospitalização de crianças com condições crônicas complexas: perspectivas e desafios. Rio de Janeiro: Eldorado. https://ler.amazon.com.br/?asin=B07YSNCL36

Carvalho Neto, F. J. d., Oliveira, F. G. L., Fontes, J. H., Neves, I. D. S., Azevedo, J. V. R. d., Vieira Júnior, D. N., Nascimento, J. M. F. d., \& Nogueira Bastos, S. N. M. A. (2020). Tecnologia educacional sobre descarte domiciliar de medicamentos. Revista de Enfermagem UFPE on line, 14. https://doi.org/10.5205/1981-8963.2020.244267

Dias, E. S. M., Rodrigues, I. L. A., Miranda, H. R., \& Corrêa, J. A. (2018). Roda de conversa como estratégia de educação em saúde para a enfermagem / Conversation wheel as education strategy in health for nursing. Revista de Pesquisa: Cuidado é Fundamental Online, 10(2). https://doi.org/10.9789/21755361.2018.v10i2.379-384

Estrela, C. (2018). Metodologia Científica: Ciência, Ensino, Pesquisa. Editora Artes Médicas.

Faleiros, F., Cucick, C. D., Silva Neto, E. T. d., Rabeh, S. A. N., Favoretto, N. B., \& Käppler, C. (2019). Desenvolvimento e validação de vídeo educativo para autocateterismo vesical intermitente limpo. Revista Eletrônica de Enfermagem, 21. https://doi.org/10.5216/ree.v21.53973

Ferreira, A. D. S., \& Abrahão, A. L. (2020). Produção do cuidado e a Educação Permanente na estratégia Saúde da Família: uma roda de conversa. Debates em Educação, 12(27). https://doi.org/10.28998/2175-6600.2020v12n27p306-315

Galvão, T. F., Pansani, T. d. S. A., \& Harrad, D. (2015). Principais itens para relatar Revisões sistemáticas e Meta-análises: A recomendação PRISMA. Epidemiol. Serv. Saúde, 24(2), 335-342. https://www.scielo.br/j/ress/a/TL99XM6YPx3Z4rxn5WmCNCF/?format=pdf\&amp;lang=pt

Gaudreau, P. A., Greenlick, H., Dong, T., Levy, M., Hackett, A., Preciado, D., Zalzal, G., \& Reilly, B. K. (2016). Preventing Complications of Pediatric Tracheostomy Through Standardized Wound Care and Parent Education. JAMA Otolaryngology-Head \& Neck Surgery, 142(10). 10.1001/jamaoto.2016.1803

Góes, F. G. B., \& Cabral, I. E. (2017). A alta hospitalar de crianças com necessidades especiais de saúde e suas diferentes dimensões [Hospital discharge in children with special health care needs and its different dimensions]. Revista Enfermagem UERJ, 25. https://doi.org/10.12957/reuerj.2017.18684

Kirchchoff, B. R. d. B., Diogo, P. F. T., Grigol, A. M., Mendes, J. S., \& Schultz, L. F. (2020). A vivência do cuidador familiar da criança em uso de traqueostomia no domicílio. Revista da Sociedade Brasileira de Enfermeiros Pediatras, 20(1), 6-12. https://doi.org/10.31508/1676-3793202000002

Lengruber, M. R., Macedo, E. C., Paula, D. G. d., Brendim, M. P., Cunha, K. C., Mendes, L. E. B., Ramos, M. N. P., \& Oliveira Junior, M. P. d. (2021). Elaboração e desenvolvimento de vídeo educacional em saúde "Conhecendo a gastrostomia". Research, Society and Development, 10(3). https://doi.org/10.33448/rsd-v10i3.13060

Martins, J. C. A., Mazzo, A., Baptista, R. C. N., Coutinho, V. R. D., Godoy, S. d., Mendes, I. A. C., \& Trevizan, M. A. (2012). A experiência clínica simulada no ensino de enfermagem: retrospectiva histórica. Acta Paulista de Enfermagem, 25(4), 619-625. https://doi.org/10.1590/s0103-21002012000400022

McDonald, J., McKinlay, E., Keeling, S., \& Levack, W. (2016). Becoming an expert carer: the process of family carers learning to manage technical health procedures at home. Journal of Advanced Nursing, 72(9), 2173-2184. https://doi.org/10.1111/jan.12984

McPherson, M., Arango, P., Fox, H., Lauver, C., McManus, M., Newacheck, P. W., Perrin, J. M., Shonkoff, J. P., \& Strickland, B. (1998). A New Definition of Children With Special Health Care Needs. Pediatrics, 102(1), 137-139. https://doi.org/10.1542/peds.102.1.137

Mendes, E. V. (2012). O cuidado das condições crônicas na atenção primária à saúde: o imperativo da consolidação da estratégia da saúde da família. Organização Pan-Americana da Saúde. https://bvsms.saude.gov.br/bvs/publicacoes/cuidado_condicoes_atencao_primaria_saude.pdf

Miranda, F. B. G., Mazzo, A., \& Pereira Junior, G. A. (2018). Uso da simulação de alta fidelidade no preparo de enfermeiros para o atendimento de urgências e emergências: revisão da literatura. Sci. med., 28(1). https://doi.org/10.15448/1980-6108.2018.1.28675

Moreira, M. C. N., Albernaz, L. V., Sá, M. R. C. d., Correia, R. F., \& Tanabe, R. F. (2017). Recomendações para uma linha de cuidados para crianças e adolescentes com condições crônicas complexas de saúde. Cadernos de Saúde Pública, 33(11). https://doi.org/10.1590/0102-311x00189516

Moura, E. C. de, Moreira, M. C. N., Menezes, L. A., Ferreira, I. A., \& Gomes, R. (2017). Complex chronic conditions in children and adolescents: hospitalizations in Brazil, 2013. Ciência \& Saúde Coletiva, 22(8), 2727-2734. https://doi.org/10.1590/1413-81232017228.01992016

Nascimento, J. D. S. G., Nascimento, K. G. d., Regino, D. D. S. G., Alves, M. G., Oliveira, J. L. G. d., \& Dalri, M. C. B. (2021). Simulação clínica: construção e validação de roteiro para o Suporte Básico de Vida no adulto. Revista de Enfermagem da UFSM, 11. https://doi.org/10.5902/2179769254578

Nazario, A. P., Lima, V. F. d., Fonseca, L. M. M., Leite, A. M., \& Scochi, C. G. S. (2021). Development and evaluation of an educational video for families on the relief of acute pain in babies. Revista Gaúcha de Enfermagem, 42. https://doi.org/10.1590/1983-1447.2021.20190386

Oliveira, N. L. L. de, Barbosa, E. M. G., Pitombeira, M. G. V., Chaves, E. M. C., \& Carvalho, R. E. F. L. d. (2020). Tecnologia educativa para cuidadores de crianças e adolescentes dependentes de cuidados especiais no domicílio. Revista Eletrônica de Enfermagem, 22. https://doi.org/10.5216/ree.v22.56051

Oliveira Neto, A. V. de, \& Borges Dias, M. (2014). Atenção Domiciliar no Sistema Único de Saúde (SUS): o que representou o Programa Melhor em Casa? Divulgação em saúde para debate, (51), 43-57. http://cebes.org.br/site/wp-content/uploads/2014/12/Divulgacao-51.pdf

Orlandin, L., Mazzo, A., Meska, M. H. G., Jorge, B. M., Cotta Filho, C. K., \& Fumincelli, L. (2017). Low-fidelity simulation for patients and caregivers in the use of lubricants in clean intermittent catheterization. International Journal of Urological Nursing, 12(1), 9-15. https://doi.org/10.1111/ijun.12155

Presado, M. H. C. V., Colaço, S., Rafael, H., Baixinho, C. L., Félix, I., Saraiva, C., \& Rebelo, I. (2018). Aprender com a Simulação de Alta Fidelidade. Ciência \& Saúde Coletiva, 23(1), 51-59. https://doi.org/10.1590/1413-81232018231.23072017 
Prickett, K., Deshpande, A., Paschal, H., Simon, D., \& Hebbar, K. B. (2019). Simulation-based education to improve emergency management skills in caregivers of tracheostomy patients. International Journal of Pediatric Otorhinolaryngology, 120, 157-161. https://doi.org/10.1016/j.jporl.2019.01.020

Ribeiro, R. P., \& Aroni, P. (2019). Standardization, ethics and biometric indicators in scientific publication: integrative review. Revista Brasileira de Enfermagem, 72(6), 1723-1729. https://doi.org/10.1590/0034-7167-2018-0283

Rossetto, V., Toso, B. R. G. d. O., Rodrigues, R. M., Viera, C. S., \& Neves, E. T. (2019). Development care for children with special health needs in home care at Paraná - Brazil. Escola Anna Nery, 23(1). https://doi.org/10.1590/2177-9465-ean-2018-0067

Rivas, E., Peña, M., \& Silva, J. A. (2013). Gestion De Cuidados De Enfermeria De Los Niños Internados Con Traqueostomia. Experiencia en el CIM 62. Cambio de cánula de traqueostomía. Protocolo de entrenamiento para padres y cuidadores. Experiencia en el Hospital Garrahan. Medicina Infantil, 20(4), 360364. https://www.medicinainfantil.org.ar/images/stories/volumen/2013/xx_4_360.pdf

Rocha, E. d. M., Paes, R. A., Sthal, G. d. M., \& Souza, A. (2019). Cuidados Paliativos: Cartilha educativa para cuidadores de pacientes oncológicos. Clinical \& Biomedical Research, 39(1), 40-57. https://doi.org/10.4322/2357-9730.85741

Salbego, C., Nietsche, E. A., Teixeira, E., Girardon-Perlini, N. M. O., Wild, C. F., \& Ilha, S. (2018). Care-educational technologies: an emerging concept of the praxis of nurses in a hospital context. Revista Brasileira de Enfermagem, 71(suppl 6), 2666-2674. https://doi.org/10.1590/0034-7167-2017-0753

Santos, N. O. d., Predebon, M. L., Bierhals, C. C. B. K., Day, C. B., Machado, D. d. O., \& Paskulin, L. M. G. (2020). Development and validation a nursing care protocol with educational interventions for family caregivers of elderly people after stroke. Revista Brasileira de Enfermagem, 73(3). https://doi.org/10.1590/0034-7167-2018-0894

Silva, R. M. M. da, Oliveira Toso, B. R. G. de, Tatsch Neves, E., Oehlmeyer Nassar, P., Zilly, A., \& Silveira Viera, C. (2017). Resolutividade na atenção à criança com necessidades especiais de saúde. Revista Pesquisa Qualitativa, 5(7), 23-37. https://editora.sepq.org.br/rpq/article/view/50/61

Sobotka, S. A., Hird-McCorry, L. P., \& Goodman, D. M. (2016). Identification of Fail Points for Discharging Pediatric Patients With New Tracheostomy and Ventilator. Hospital Pediatrics, 6(9), 552-557. https://doi.org/10.1542/hpeds.2015-0277

Sousa Lima, B. de, Siqueira Lima Freitas, C., Alves Monteiro Da Silva, M., De Azevedo Mazza, V., Cordeiro Eloia, S., \& Martins da Silva, G. (2021). Vivências de familiares de crianças com deficiência. Revista Enfermagem Atual In Derme, 95(33). https://doi.org/10.31011/reaid-2021-v.95-n.33-art.911

Thrasher, J., Baker, J., Ventre, K. M., Martin, S. E., Dawson, J., Cox, R., Moore, H. M., Brethouwer, S., Sables-Baus, S., \& Baker, C. D. (2018). Hospital to Home: A Quality Improvement Initiative to Implement High-fidelity Simulation Training for Caregivers of Children Requiring Long-term Mechanical Ventilation. Journal of Pediatric Nursing, 38, 114-121. https://doi.org/10.1016/j.pedn.2017.08.028

Viana, I. d. S., Silva, L. F. d., Cursino, E. G., Conceição, D. S. d., Goes, F. G. B., \& Moraes, J. R. M. M. d. (2018). Encontro educativo da enfermagem e da família de crianças com necessidades especiais de saúde. Texto \& Contexto - Enfermagem, 27(3). https://doi.org/10.1590/0104-070720180005720016

Zocche, D.A.A., Argenta Zanatta, E., Adamy, E. K., Vendruscolo, C., \& de Lima Trindade, L. (2020). Protocolo para revisão integrativa: caminho para busca de evidências. In E. Teixeira (Ed. 2ª), Desenvolvimento de Tecnologias Cuidativo-Educacionais (pp. 237-249). Moriá. 